\title{
Beyond NMF: Advanced Signal Processing and Machine Learning Methodologies for Hyperspectral Analysis in EELS
}

Jordan Hachtel ${ }^{1}$, Nikolay Borodinov ${ }^{2}$, Kevin Roccapriore ${ }^{2}$, Shin Hum Cho ${ }^{3}$, Progna Banerjee ${ }^{4}$, Delia Milliron $^{5}$, Olga Ovchinnikova ${ }^{6}$, Maxim Ziatdinov $^{6}$, Sergei Kalinin ${ }^{7}$ and Rama Vasudevan ${ }^{2}$

${ }^{1}$ Center for Nanophase Materials Sciences, Oak Ridge National Laboratory, United States, ${ }^{2}$ Oak Ridge National Laboratory, United States, ${ }^{3}$ Samsung Electronics, United States, ${ }^{4}$ Argonne National Laboratory , United States, ${ }^{5}$ University of Texas at Austin, United States, ${ }^{6}$ Computational Sciences and Engineering Division, Oak Ridge National Laboratory, Oak Ridge, Tennessee, United States, ${ }^{7}$ Center for Nanophase Materials Sciences, Oak Ridge National Laboratory, Oak Ridge, Tennessee, United States

Component separation techniques such as non-negative matrix factorization (NMF) have found a permanent home in the electron energy-loss spectroscopy (EELS) community due to their ability to separate overlapped signals in a hyperspectral dataset. Especially in the field of nanoplasmonic, where NMF components can directly represent physical plasmon modes in simple systems [1]. While NMF has only been extensively used in EELS over the last decade, it has been an established technique in the signal processing community for much longer [2]. We believe there is significant potential application to porting other established signal processing techniques to EELS hyperspectral analysis. Here, we will discuss two such techniques: a data fusion methodology called pan-sharpening and unsupervised component separation methodology using a type of machine-learning network called an autoencoder.

Pan-sharpening is a class of methodologies developed for satellite imaging, where two datasets with different beneficial properties are combined into one dataset with the beneficial properties of both. For the application in EELS, we acquire two hyperspectral datasets on the same region: one with a large number of pixels (and hence high spatial resolution) and one with a long acquisition time for each spectrum (and hence high spectral fidelity). We then perform an NMF decomposition on the high spectral fidelity dataset and use the spectral endmembers to solve for abundance maps in the high spatial resolution dataset, creating a single dataset with both high spatial resolution and high spectral fidelity [3], as shown in Figure 1 .

For the autoencoder component separation, it is important to note that NMF itself is a form of unsupervised component separation. While for simple systems, NMF is extremely effective at separating and isolating individual physical mechanisms, for complex systems the interpretability of the components can become highly challenging. In our approach we use the autoencoder to convert the entire hyperspectral dataset into a latent space, where all spectra in the dataset can be represented by Cartesian coordinates in latent space [4]. By reducing the dimensionality of a hyperspectral dataset in this manner analysis can be performed directly in latent space using distance metrics, such as k-means clustering or Gaussian mixture modeling (shown in Fig. 2). The latent-space analysis labels the individual pixels in the dataset but does not alter them, meaning physical mechanisms are separated spatially without the EELS signal at the labeled pixels being altered. This provides an excellent alternative to NMF to unmix components in complex systems. 

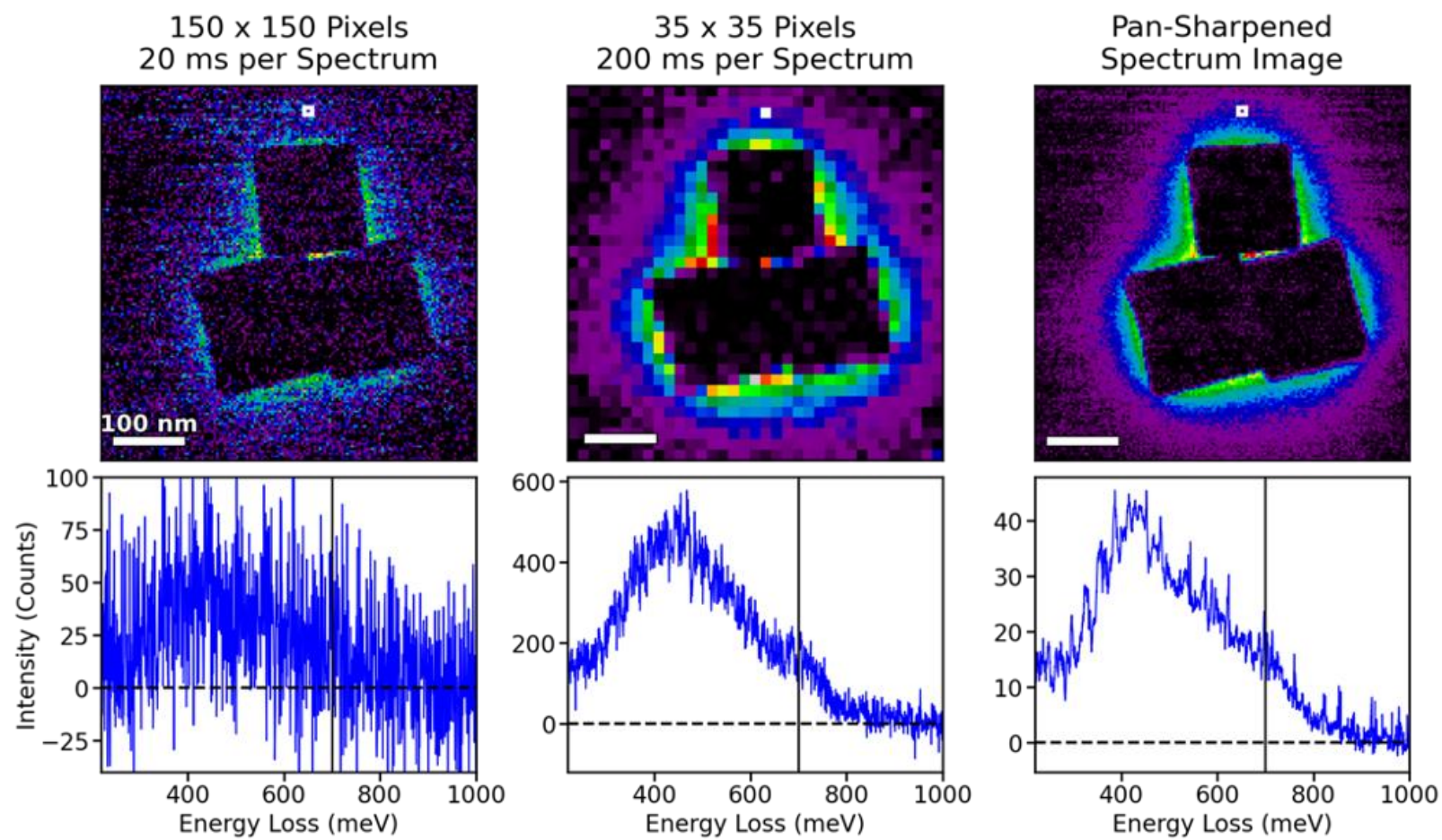

Figure 1. Figure 1. Example of pan-sharpening. We combine NMF decompositions in a high spatial resolution dataset with a high spectral fidelity dataset to achieve one dataset with the beneficial properties of both. The images are slices of the hyperspectral dataset at $700 \mathrm{meV}$ (marked with a blac line in the spectra) and the spectra are from the white squares in the slices.
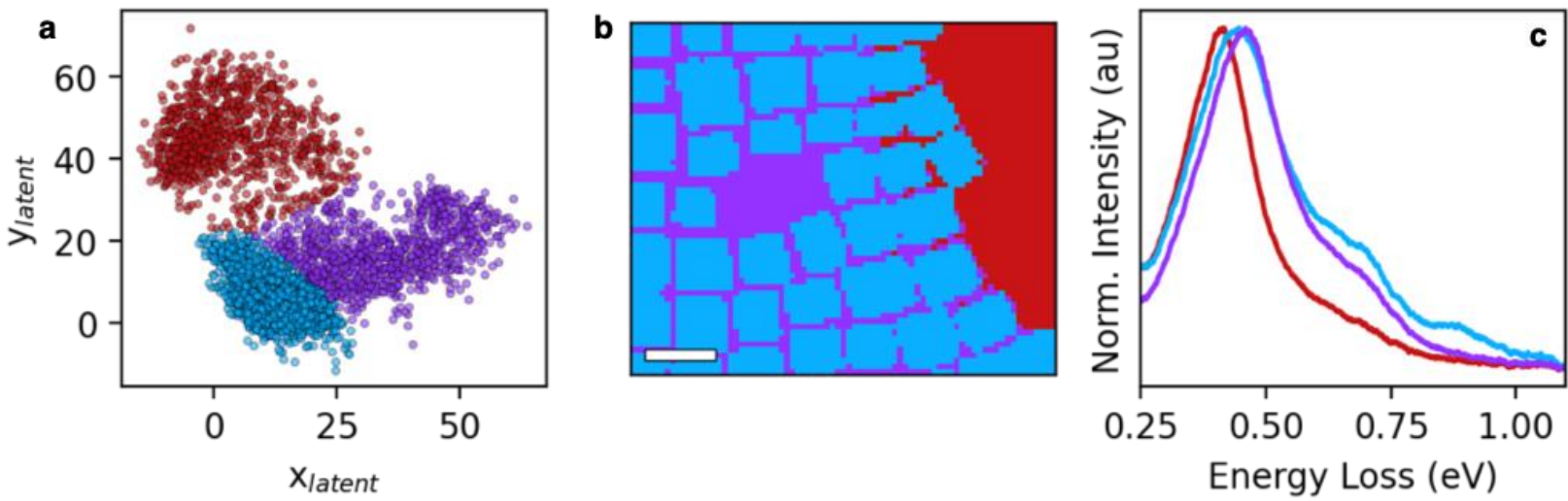

Figure 2. Figure 2. Analysis in latent space generated by autoencoder. (a) A hyperspectral dataset is encoded into latent space. Each point represents a spectrum in the dataset, and we use Gaussian mixture modeling to break the dataset into three labels. (b) The original dataset can now be shown in terms of the localization of the three labels. (c) The average spectra from the labeled pixels in (b). 


\section{References}

[1] O Nicoletti et al., Nature $\mathbf{5 0 2}$ (2013), p. 80

[2] WH Lawton and EA Sylvestre, Technometrics 13 (1971), p. 617

[3] N Borodinov et al., J. Chem. Phys. 154 (2021), p. 014202

[4] SV Kalinin et al., arXiv (2020) arXiv:2009.08501

[5] Research conducted at the Center for Nanophase Materials Sciences, which is a DOE Office of Science User Facility. Work was performed, in part, using instrumentation within ORNL's Materials Characterization Core provided by UT-Battelle, LLC, under Contract No. DE-AC05- 00OR22725 (J.C.I.) with the DOE, and sponsored by the Laboratory Directed Research and Development Program of Oak Ridge National Laboratory, managed by UT-Battelle, LLC, for the U.S. Department of Energy. 